\title{
Attachment and Parenting in Adult Patients with Anxiety Disorders
}

\author{
Angelo Picardi ${ }^{1, *}$, Emanuele Caroppo ${ }^{2}$, Elisa Fabi $^{3}$, Serena Proietti ${ }^{4}$, Giancarlo Di Gennaro ${ }^{5}$, Giulio \\ Nicolò Meldolesi, ${ }^{5,6}$ and Giovanni Martinotti ${ }^{7,8}$
}

\author{
${ }^{I}$ Mental Health Unit, Centre of Epidemiology, Surveillance and Health Promotion, Italian National Institute of Health, \\ Rome, Italy \\ ${ }^{2}$ RM-B Local Health Unit, Department of Mental Health, Rome, Italy \\ ${ }^{3}$ Department of Neurology and Psychiatry, Policlinico Umberto I, Sapienza University of Rome, Italy \\ ${ }^{4}$ Department of Psychiatry and Psychology, Catholic University of Rome, Italy \\ ${ }^{5}$ Epilepsy Surgery Unit, IRCCS Neuromed, Pozzilli, Italy \\ ${ }^{6}{ }^{\prime}$ Neurone' Foundation for Research in Neuropsychobiology and Clinical Neurosciences, Rome, Italy \\ ${ }^{7}$ Neuropsychiatric Facility 'Villa Maria Pia', Rome, Italy \\ ${ }^{8}$ Department of Neuroscience and Imaging, University "G.d'Annunzio", Chieti, Italy
}

\begin{abstract}
Background: The literature suggests that dysfunctional parenting and insecure attachment may increase risk of anxiety-related psychopathology. This study aimed at testing the association between anxiety disorders, attachment insecurity and dysfunctional parenting while controlling for factors usually not controlled for in previous studies, such as gender, age, and being ill.

Methods: A sample of 32 non-psychotic inpatients with SCID-I diagnosis of an anxiety disorder, either alone or in comorbidity, was compared with two age- and sex-matched control groups consisting of 32 non-clinical participants and 32 inpatients with drug-resistant epilepsy. Study measures included the Experience in Close Relationships questionnaire (ECR) and the Parental Bonding Instrument (PBI).

Results: The patients with anxiety disorders scored significantly higher on attachment-related anxiety and avoidance than patients with drug-resistant epilepsy and non-clinical participants. These findings were independent of comorbidity for mood disorders. ECR scores did not differ among diagnostic subgroups (generalized anxiety disorder, panic disorder, other anxiety disorders). Patients with anxiety disorders scored significantly lower on PBI mother's care and borderline significantly lower on PBI father's care than patients with drug-resistant epilepsy.

Conclusions: Although limitations such as the relatively small sample size and the cross-sectional nature suggest caution in interpreting these findings, they are consistent with the few previous adult studies performed on this topic and corroborate Bowlby's seminal hypothesis of a link between negative attachment-related experiences, attachment insecurity, and clinical anxiety. Attachment theory provides a useful theoretical framework for integrating research findings from several fields concerning the development of anxiety disorders and for planning therapeutic interventions.
\end{abstract}

Keywords: Attachment, parenting, stress, emotion regulation, anxiety, epilepsy.

\section{INTRODUCTION}

Anxiety disorders are the most prevalent class of mental disorders, and they carry a substantial burden on patients. Recent estimates suggest that 61.5 millions of people in the European Union are affected by an anxiety disorder over a 12-month period; also, disorders such as panic disorder and obsessive-compulsive disorder rank among the 15 leading

\footnotetext{
*Address correspondence to this author at the Italian National Institute of Health, Centre of Epidemiology, Surveillance and Health Promotion, Mental Health Unit, Viale Regina Elena, 299 - 00161 Rome, Italy;

Tel: +39 06 49904200; Fax: +3906 49904182;

E-mail: angelo.picardi@iss.it
}

psychiatric and neurological causes of disease burden, expressed as the number of years lost due to ill-health, disability or early death [1].

A variety of factors contribute to the development of anxiety disorders. Risk factors include female gender, poverty, family history of anxiety, early adversity, and negative life events $[2,3]$. The strong familial aggregation of anxiety disorders may be due to genetic influences, family environment factors, or both. Genetically informative studies that can disentangle genetic from environmental contributions in the familial transmission of anxiety disorders found evidence of a moderate genetic heritability [4], which suggests that 
family environmental factors also play a role in increasing vulnerability to anxiety.

Given the key role of attachment in the development of emotion regulation [5] and the link between dysfunctional affect regulation and anxiety, factors such as dysfunctional parenting and insecure attachment may confer risk for the development of anxiety-related psychopathology [6]. Indeed, stressful experiences, physiological stress regulation, and attachment relationships are intrinsically linked, as the activation of the attachment system invariably follows the early stages of detecting and processing fear-related cues and anxiety states. As affect regulation strategies are mainly acquired in the interpersonal context of early attachment relationships and persist into adulthood, they are relevant for the understanding of the nature and development of anxiety disorders [7].

In the framework of attachment theory [8], the term 'attachment' is used to indicate the emotional, cognitive, and behavioural processes implicated in the development and maintenance of intimate social bonds. While all humans share a basic disposition to form and maintain intimate bonds that are essential for survival and good health from early childhood to old age, each individual shows a distinct attachment style which reflects individual differences in affect regulation and in perceptions of and beliefs about self and significant others. Two major dimensions, called attachment-related anxiety (about abandonment or insufficient love) and avoidance (of intimacy and emotional expression), underlie adult attachment style and conceptually relate to similar dimensions that can be observed in infants during a stressful laboratory procedure, called the 'Strange Situation', which involves separations from mother, exposure to an adult stranger, and reunions with mother [9]. Attachmentrelated anxiety corresponds to continuous coding scales measuring infant distress and anger, whereas attachmentrelated avoidance relates to scales measuring avoidance of mother, lack of closeness to mother, and less distress in front of the stranger during mother's absence [10]. High levels of attachment-related anxiety characterize insecure attachment patterns named as ambivalent or resistant in children and preoccupied in adults, while elevated levels of attachmentrelated avoidance characterize attachment patterns named as avoidant in children and dismissing or dismissive in adults. As far as romantic attachment is concerned, individuals with high attachment-related anxiety tend to be preoccupied with their romantic relationships, to feel unappreciated, and to worry about being abandoned or not loved enough. Adults with high attachment-related avoidance have difficulty trusting or depending on their partner, are reluctant to ask him or her for support, and feel uneasy with emotional closeness and intimacy.

Parenting style can be defined as a constellation of attitudes towards the child that, taken together, create an emotional climate in which the parents' behaviours (both the specific, goal-directed behaviours commonly referred to as parental practices, and non-goal directed behaviours, such as gestures, changes in tone of voice, or spontaneous display of emotion) are expressed. In the work of researchers and scholars with different theoretical backgrounds, such as psychodynamic, behavioural, and social learning theorists, there is a surprising agreement on the dimensions underlying parenting style. The dimensions of nurturance and control feature prominently in this literature, although different names are often used, such as care, acceptance, love, or warmth for the first dimension, and dominance or restrictiveness for the second one [11]. Optimal parenting is typically viewed as the combination of high care and low control [12].

The studies exploring the association between attachment and anxiety in childhood and adolescence showed a moderate association between attachment insecurity, especially anxious/ambivalent attachment, and child and adolescent anxiety [13]. The studies investigating the association between parenting style and child anxiety yielded mixed results, possibly due to different study designs and measurement contexts and methods, with stronger and more reliable associations generally found for parental overcontrol as compared with parental rejection [14].

Relatively few studies comparing patients with anxiety disorders with non-psychiatric samples with regard to parenting and attachment were performed in adults. All these studies reported an association between attachment insecurity and anxiety [15-18]. Negative attachment-related experiences such as unfavorable parental attitudes were also found to be associated with disorders such as panic disorder [19, 20] and generalized anxiety disorder [21].

Most previous studies on the association between anxiety disorders and attachment insecurity or dysfunctional parenting did not control for the possible confounding by factors such as demographic variables or the state of being unhealthy. In the present study, we aimed at testing the hypothesis that anxiety disorders are associated with attachment insecurity or dysfunctional parenting independently of the possible influence of demographic variables or the state of being unhealthy. Demographic variables were controlled for by matching, while the state of being unhealthy was controlled for by including not only a non-clinical control group, but also a control group affected by a chronic disease. To this purpose, we compared the scores on two well-validated measures of parenting and attachment style obtained by a sample of inpatients diagnosed with an anxiety disorder by means of a standardized psychiatric interview with the scores obtained by two age- and sex-matched control groups consisting of non-clinical participants and in patients with epilepsy, respectively.

\section{METHODS}

\section{Participants}

The patients with anxiety disorders were recruited in a private psychiatric facility located in Rome and accredited by the Italian National Health Service. All adult patients without dementia or severe cognitive impairment as clinically determined who were consecutively admitted to the inpatient ward over a three-year enrollment period were invited to participate in a research project focusing on individual differences and psychopathology. All patients were given the opportunity to ask further questions about the research project, and those who accepted were asked to sign a statement of informed consent. 
Within three days from admission, the participants were administered several self-report measures, including the Experience in Close Relationships questionnaire (ECR) [10] and the Parental Bonding Instrument (PBI) [12], as well as a number of clinician-rated instruments, among which the Structured Clinical Interview for DSM-IV Axis I (SCID-I) [22]. All non-psychotic patients who were diagnosed with an anxiety disorder, either alone or in comorbidity, were considered for inclusion in this study. Of 35 eligible patients, only three did not return a completed ECR or PBI. Therefore, the study sample consists of 32 patients (12 males and 20 females, mean age $43.16 \pm 12.77$ years) of whom 13 were diagnosed with panic disorder, 12 with generalized anxiety disorder, three with social anxiety disorder, two with obsessive-compulsive disorder, one with post-traumatic stress disorder, and one with anxiety disorder not otherwise specified. Sixteen patients $(50 \%)$ had a comorbid mood disorder.

Two comparison groups, both matched for age and sex, were selected. The first was a standard non-clinical control group that included 32 participants (12 males and 20 females, mean age $43.16 \pm 12.76$ years) recruited in the community who completed the ECR and PBI as part of an ongoing study on individual differences and emotion regulation. In order to control for the presence of illness and for hospitalization, we also included another comparison group consisting of 32 inpatients with drug-resistant epilepsy who completed the ECR and PBI as part of a multidisciplinary presurgical assessment protocol at the Epilepsy Surgery Unit of the Neuromed-IRCCS Neurological Institute, located in Pozzilli, a small town about 100 miles from Rome, Italy. Due to the usually young age of this patient population, we could not accomplish perfect matching for age in this group; nevertheless, the demographic characteristics provided a reasonable match for patients with anxiety disorders as the neurological control group included 12 males and 20 females, with a mean age of $37.72 \pm 8.61$ years.

\section{Assessment Instruments}

The ECR is a self-report instrument consisting of 36 items, each scored on a seven-point scale. The instrument provides scores on two dimensions, named 'Anxiety' and 'Avoidance'. Each scale consists of 18 items, some of which are reverse-keyed, and is scored by summing the scores for all the items constituting the scale. Scores on each ECR scale can range from 18 to 126 , with higher scores indicating greater insecurity with respect to the attachment dimension being measured. Individuals scoring high on the Anxiety scale tend to be preoccupied with their romantic relationships, to worry about being abandoned, to desire a high level of closeness to their partner, and to ask the partner for more feeling and commitment. Individuals scoring high on the Avoidance scale tend to avoid emotional closeness and intimacy, to feel uncomfortable opening up to or depending on their partner, and to be reluctant to ask their partner for comfort, advice, or help. We used the validated Italian version of the instrument [23].

The PBI is a self-completed questionnaire consisting of 25 items, scored on a 4-point Likert scale indicating the degree of agreement with the item statement. Twelve items refer to empathy and care ('Care') and 13 to excessive control and protection ('Overprotection') that subjects recall to have received from either their mother (maternal form) or father (paternal form) during their first 16 years of life. Therefore, the Care scale measures the degree to which the parent was empathic and caring or cold and indifferent, while the Overprotection scale measures the extent to which the parent was intrusive and infantilizing or, in contrast, fostered independence in the subject.

The SCID-I is a clinician-administered interview that covers most Axis I disorders and is regarded as the standard for making DSM-IV diagnoses. Several studies showed its superior validity over standard clinical interviews at intake episode $[24,25]$. We used the official Italian version of the instrument [26]. The SCID-I was administered by three experienced raters (a psychiatrist, a clinical psychologist and a senior resident in psychiatry) who received specific training in administering and scoring the instruments prior to the study. The interviewers based their ratings on all sources of information available, including not only patients' answers and clinical observation, but also referral notes, medical records, and reports of significant others. The inter-rater reliability was assessed on three occasions during the study period using conjoint interviews of psychiatric patients in which one rater interviewed the patient and the other raters listened to the interview and did not ask clarifying questions. Overall, the reliability of the SCID-I as measured by the kappa coefficient ranged from .75 to .91 for the more common diagnoses (psychotic, mood, anxiety, and substance use disorders).

\section{Statistical Analysis}

All analyses were performed with SPSS for Mac, version 20.0. All statistical tests were two-tailed, with alpha set at 0.05 . First, a descriptive analysis was used to study the frequency distribution of all variables of interest. Then, analysis of variance was used to test for differences between study groups in ECR and PBI scores. Tukey's post-hoc test was used for pairwise comparisons.

\section{RESULTS}

Table 1 shows the mean scores on the study measures by group.

ECR scores were available for 31,29 , and 31 participants in the psychiatric, neurological, and non-clinical group, respectively. The patients with anxiety disorders scored significantly higher on attachment-related anxiety than patients with drug-resistant epilepsy $(\mathrm{p}=0.011)$ and non-clinical participants $(p=0.003)$. Comorbidity for mood disorders did not affect the results as attachment-related anxiety mean scores were 82.7 and 82.9 in patients with and without a comorbid mood disorder, respectively.

Patients with anxiety disorders also scored significantly higher on attachment-related avoidance as compared with patients with drug-resistant epilepsy $(\mathrm{p}=0.003)$ and nonclinical participants $(\mathrm{p}=0.001)$. While attachment-related avoidance mean scores were slightly higher among patients with a comorbid mood disorder (62.9) as compared with patients without mood comorbidity (57.5), the differences between patients with anxiety disorders and both comparison groups remained significant $(\mathrm{p}=0.029$ and $\mathrm{p}=0.012$ for the 
Table 1. ECR and PBI Scores (mean \pm SD) by Group

\begin{tabular}{|l|c|c|c|}
\hline & Patients with Anxiety Disorders & Patients with Drug-Resistant Epilepsy & Non-Clinical Participants \\
\hline \hline Attachment-related Anxiety & $82.8 \pm 23.8^{* *}$ & $66.4 \pm 20.8$ & $64.3 \pm 19.0$ \\
\hline Attachment-related Avoidance & $60.1 \pm 24.7^{* *}$ & $42.0 \pm 21.3$ & $40.0 \pm 14.2$ \\
\hline Mother's Care & $21.0 \pm .7 .3^{*}$ & $27.6 \pm 6.2$ & $24.1 \pm 7.4$ \\
\hline Mother's Overprotection & $22.6 \pm .7 .4$ & $19.2 \pm .6 .2$ & $19.3 \pm .8 .4$ \\
\hline Father's Care & $20.5 \pm .8 .7$ & $26.6 \pm .7 .4$ & $20.4 \pm .4 .5$ \\
\hline Father's Overprotection & $16.8 \pm .5 .9$ & $16.9 \pm .7 .7$ & $18.9 \pm .5 .5$ \\
\hline
\end{tabular}

* significantly lower as compared with patients with drug-resistant epilepsy

** significantly higher as compared both with patients with drug-resistant epilepsy and non-clinical participants

neurological and non-clinical control group, respectively) when the analysis was repeated excluding patients with a comorbid mood disorder.

In order to examine the possible association between specific diagnostic categories and attachment style, we subdivided the psychiatric group into three categories (generalized anxiety disorder, panic disorder, other anxiety disorders). No differences in ECR scores between these diagnostic subgroups were found.

Mother's and Father's PBI scores were available for 17 and 12 patients with anxiety disorders, respectively. In order to maintain the statistical control for age and sex, only the comparison subjects that were specifically paired with the psychiatric patients who returned a completed Mother's and Father's PBI were included in the respective analysis of variance model. The patients with anxiety disorders scored significantly lower on mother's care than patients with drugresistant epilepsy $(p=0.023)$. They also scored lower than non-clinical participants, although the difference did not reach statistical significance $(\mathrm{p}=0.41)$. No differences between group in mother's overprotection scores were found. There was a trend for patients with anxiety disorders to score lower on father's care as compared with patients with epilepsy, though the results did not reach significance $(p=0.107)$. No differences between groups in father's overprotection scores were observed.

\section{DISCUSSION}

Already in the Seventies, Bowlby postulated a link between attachment insecurity and anxiety. In his view, adult anxiety may be rooted in childhood experiences that leave a child uncertain about the availability of a protective figure in times of trouble [27]. Subsequently, many authors [28-30] described ways in which attachment insecurity may contribute to the development of anxiety disorders. Difficulties with separation, intolerance of uncertainty, and the tendency to process events as out of one's control are some of the mechanisms that have been proposed to explain the link between attachment insecurity and anxiety.

Previous studies on adults reported an association between anxiety disorders and attachment-related anxiety. Patients with obsessive-compulsive disorder showed greater relationship anxiety than controls [17]. In another study on a mixed psychiatric sample, the patients with panic disorder and obsessive-compulsive disorder scored higher on attachment-related anxiety as compared with healthy individuals [31]. Recently, attachment anxiety was found to be significantly higher in individuals with obsessive-compulsive disorder than in healthy controls, even when controlling for depression [18]. Consistently with theoretical expectations [7] and with these previous studies, we found significantly higher levels of attachment-related anxiety among patients with anxiety disorders as compared with both healthy controls and patients with drug-resistant epilepsy. We also found an association of similar strength between anxiety disorders and attachment-related avoidance. Importantly, these associations were independent of depression, as they were found both in patients with and without a comorbid mood disorder.

The studies investigating the association between attachment and anxiety symptoms or disorders in childhood and adolescence suggest that ambivalent or preoccupied attachment has a stronger relation with anxiety disorders than avoidant attachment or attachment insecurity in general [13]. Also, a recent sophisticated theoretical model suggested that the characteristic up-regulation of stress reactivity observed in individuals high in attachment anxiety may be an important risk factor for the development of anxiety disorders [7]. While the hyperactivating strategies to regulate affect that characterize individuals with high attachment anxiety may bear a stronger relation with anxiety disorders as compared with the deactivating strategies that characterize individuals with high attachment avoidance, several authors also pointed to theoretically consistent ways that could account for a link between avoidant attachment and anxiety. It has been suggested that whereas children with high attachment anxiety may develop separation anxiety as they are used to continuously request the caregivers' attention, children with high attachment avoidance may develop both social phobia and separation anxiety as they are used to cope with the experience of rejection [32]. As anxiety disorders are characterized by a combination of fear and avoidance, it has also been proposed that hyperactivating strategies are associated with anxiety disorders in which fear predominates, whereas deactivating stretegies are associated with anxiety disorders in which avoidance predominates [33]. It should be acknowledged that, despite being adaptive in childhood, both insecure strategies have a detrimental impact on interpersonal 
functioning in later life. Interpersonal stress buffering processes such as social support are less effective in insecurely attached individuals, which may in turn increase the risk of developing an anxiety disorder. Indeed, it seems plausible that both types of insecurity are linked to anxiety as they both imply negative expectations about the availability or responsiveness of significant others as well as decreased capacity to regulate negative emotions. Consistently with our findings, other previous studies performed on adults found an association between high levels of avoidance and anxiety disorders. In a study, single patients with obsessive-compulsive disorder reported more discomfort with dependence than did healthy controls [17]. In another study, patients with panic disorder and obsessive-compulsive disorder scored higher on attachment-related avoidance as compared with healthy individuals [31].

An inspection of individual scores showed that the finding of higher mean scores on both ECR scales in patients with anxiety disorders as compared with the control groups arose from the presence of high scores (20 or more points above the mean score of healthy controls) on both attachment-related anxiety and avoidance in about one third of patients, with a few patients scoring high on either avoidance or anxiety alone. High levels of both attachment avoidance and anxiety suggest the presence, at least in some patients, of a fearful avoidant attachment style, which shares many similarities with the concept of disorganized attachment [34, 35]. Other studies suggested a link between attachment disorganization and anxiety. Pioneer studies reported a high prevalence of unresolved attachment states of mind in adult patients with anxiety disorders $[15,36]$, while a recent study on late adolescents found that the presence of anxiety disorders and comorbid conditions was associated with higher levels of attachment disorganization [16].

With one exception [17], previous studies found that patients with anxiety disorders reported lower parental care. Compared to subjects with no history of psychiatric disorders, patients with panic disorder described the attitude of their parents as more restricting and providing less loving care and attention [19]. In another study, patients with panic disorder reported their parents to be significantly less caring than did the control group [20]. A study on patients with generalized anxiety disorder found that, compared to control participants, the patients reported experiencing less maternal love in childhood, greater maternal rejection/neglect, and more maternal role-reversal/enmeshment [21]. In the present study, fewer group differences were found in parenting as compared with attachment. The relatively less robust relationship observed between parenting and anxiety compared to attachment and anxiety may be ascribed to low statistical power due to missing PBI data, or to a biasing effect of attachment security on recollections of parental rearing. The findings were nevertheless consistent with those observed for attachment style, as patients with anxiety disorders reported to a greater extent negative attachment-related experiences such as low maternal care.

This study has several limitations. First, the sample size was relatively small, which reduced statistical power. While this did not prevent us to detect significant differences in attachment style between groups, it may have obscured some differences between groups in PBI scores. Also, the crosssectional nature of the study precludes causal inferences. A further limitation is the inclusion of prevalent rather than incident cases of anxiety disorders. Although individual differences in attachment security are fairly stable across the lifespan $[37,38]$ and there is evidence of absolute and relative stability for ECR scores [39], it is impossible to rule out that the higher levels of attachment insecurity observed in patients with anxiety disorders represents a consequence of having being unhealthy for months or years. Nevertheless, the use of a control group consisting of patients with a chronic, severe disease often accompanied by high levels of depressive and anxiety symptoms [40] mitigates this limitation. Finally, it should be acknowledged that our findings are limited to adult attachment style as measured by self-report measures of adults' perceptions and views about themselves and others in romantic relationships, which are not interchangeable with interview-based measures of adults' current state of mind and coherence of discourse regarding childhood attachment experiences with parents [41]. Therefore, further studies using interview-based measures of attachment would be important to complement our study.

With these limitations in mind, this study corroborated the hypothesis that attachment insecurity is associated with anxiety disorders. As it provides a developmental account of both normative and maladaptive stress regulation, attachment theory is well suited to integrate the variety of findings that are emerging from the various fields investigating stress and anxiety disorders [7]. It also provides a useful theoretical framework for planning therapeutic interventions, either alone or in addition to other treatment components, including medication. Indeed, authors from different theoretical perspectives, such as psychoanalysis [42-45], cognitive therapy $[28,46]$, and family therapy [47] have described how attachment theory can usefully inform the psychotherapy process. A common theme across this clinical literature is the conceptualization of the therapist as a secure base from which the patient can safely reflect on his or her experience, think about the possible contents of the minds of significant others, and explore the possibility of trying new experiences and experimenting with novel behaviours. Other common themes include providing the patient with opportunities to mourn past losses, exploring the impact of important relationships on the patient's life in the present and the past, helping the patient understand his or her active contribution to interpersonal difficulties, and working to improve the patient's emotion regulation and mentalization abilities.

Attachment-informed research in the field of anxiety disorders is still relatively scarce, and future studies are necessary to address a number of key issues. First, more specific research into the relation between certain attachment dimensions or categories and particular types of anxiety disorder is needed. Longitudinal neuroscientific developmental research would likely be necessary to elucidate the complex interactions that result in different types of anxiety disorders. Also, studies are needed to test the effectiveness of attachmentbased interventions in reducing anxiety. Finally, future stud- 
ies should also examine the complex interplay between attachment insecurity and other risk factors for anxiety, such as demographic factors, genetic influences, and individual experiences.

\section{CONFLICT OF INTEREST}

The authors confirm that this article content has no conflicts of interest.

\section{ACKNOWLEDGEMENTS}

This study was supported by the 'Neurone' Foundation for research in neuropsychobiology and clinical neurosciences, Rome, Italy.

\section{REFERENCES}

[1] Wittchen HU, Jacobi F, Rehm J, et al. The size and burden of mental disorders and other disorders of the brain in Europe 2010. Eur Neuropsychopharmacol 2011; 21: 655-79.

[2] Craske MG, Waters AM. Panic disorder, phobias, and generalized anxiety disorder. Annu Rev Clin Psychol 2005; 1: 197-225.

[3] Beesdo-Baum K, Knappe S. Developmental epidemiology of anxiety disorders. Child Adolesc Psychiatr Clin N Am 2012; 21: 45778 .

[4] Smoller JW, Block SR, Young MM. Genetics of anxiety disorders: the complex road from DSM to DNA. Depress Anxiety 2009; 26: 965-75.

[5] Mikulincer M, Shaver PR. Adult attachment and emotion regulation. In: Cassidy J, Shaver PR, Eds. Handbook of attachment: Theory, research, and clinical applications. $2^{\text {nd }}$ ed. New York: Guilford Press 1999; pp. 503-31.

[6] Esbjørn BH, Bender PK, Reinholdt-Dunne ML, Munck LA, Ollendick TH. The development of anxiety disorders: considering the contributions of attachment and emotion regulation. Clin Child Fam Psychol Rev 2012; 15: 129-43.

[7] Nolte T, Guiney J, Fonagy P, Mayes LC, Luyten P. Interpersonal stress regulation and the development of anxiety disorders: an attachment-based developmental framework. Front Behav Neurosci 2011; 5: 55 .

[8] Bowlby J. Attachment and loss. vol. 1: Attachment. New York: Basic Books, 1969.

[9] Ainsworth MDS, Blehar MC, Waters E, Wall S. Patterns of attachment: A psychological study of the Strange Situation. Hillsdale: Erlbaum, 1978.

[10] Brennan KA, Clark CL, Shaver PR. Self-report measurement of adult attachment: an integrative overview. In: Simpson JA, Rholes WS, Eds. Attachment theory and close relationships. New York : Guilford Press 2008; pp. 46-76.

[11] Darling N, Steinberg L. Parenting style as context: An integrative model. Psychol Bull 1993; 113: 487-96.

[12] Parker G, Tupling H, Brown LB. A Parental Bonding Instrument. Br J Med Psychol 1979; 52: 1-10.

[13] Colonnesi C, Draijer EM, Jan JM, et al. The relation between insecure attachment and child anxiety: a meta-analytic review. J Clin Child Adolesc Psychol 2011; 40: 630-45.

[14] McLeod BD, Wood JJ, Weisz JR. Examining the association between parenting and childhood anxiety: a meta-analysis. Clin Psychol Rev 2007; 27: 155-72.

[15] Fonagy P, Leigh T, Steele M, et al. The relation of attachment status, psychiatric classification, and response to psychotherapy. J Consult Clin Psychol 1996; 64: 22-31.

[16] Brumariu LE, Obsuth I, Lyons-Ruth K. Quality of attachment relationships and peer relationship dysfunction among late adolescents with and without anxiety disorders. J Anxiety Disord. 2012; 27(1): 116-24.

[17] Myhr G, Sookman D, Pinard G. Attachment security and parental bonding in adults with obsessive-compulsive disorder: a comparison with depressed out-patients and healthy controls. Acta Psychiatr Scand 2004; 109: 447-56.

[18] Doron G, Moulding R, Nedeljkovic M, Kyrios M, Mikulincer M, Sar-El D. Adult attachment insecurities are associated with obsessive compulsive disorder. Psychol Psychother 2012; 85: 163-78.
[19] Bandelow B, Späth C, Tichauer GA, Broocks A, Hajak G, Rüther E. Early traumatic life events, parental attitudes, family history, and birth risk factors in patients with panic disorder. Compr Psychiatry 2002; 43: 269-78.

[20] Pacchierotti C, Bossini L, Castrogiovanni A, Pieraccini F, Soreca I, Castrogiovanni P. Attachment and panic disorder. Psychopathology 2002; 35: 347-54.

[21] Cassidy J, Lichtenstein-Phelps J, Sibrava NJ, Thomas CL Jr, Borkovec TD. Generalized anxiety disorder: connections with selfreported attachment. Behav Ther 2009; 40: 23-38.

[22] First MB, Spitzer RL, Gibbon M, Williams JBW. Structured Clinical Interview for DSM-IV Axis I Disorders, Clinical Version (SCID-I CV). Washington, DC: American Psychiatric Press 1996.

[23] Picardi A, Vermigli P, Toni A, D’Amico R, Bitetti D, Pasquini P. Further evidence of the validity of the Italian version of the questionnaire 'Experiences in Close Relationships' (ECR), a self-report instrument to assess adult attachment. Ital J Psychopathol 2002; 8: 282-94.

[24] Basco MR, Bostic JQ, Davies D, et al. Methods to improve diagnostic accuracy in a community mental health setting. Am J Psychiatry 2000; 157: 1599-605.

[25] Fennig S, Naisberg-Fennig S, Craig TJ, Tanenberg-Karant M, Bromet EJ. Comparison of clinical and research diagnoses of substance use disorders in a first-admission psychotic sample. Am J Addict 1996; 5: 40-8.

[26] Mazzi F, Morosini P, de Girolamo G, Lussetti M, Guaraldi GP. SCID-I CV: Intervista clinica strutturata per i disturbi dell'asse I del DSM-IV, versione clinica. Firenze: Organizzazioni Speciali 2000.

[27] Bowlby J. Attachment and loss: vol. 2. Separation: anxiety and anger. New York: Basic Books, 1973.

[28] Guidano VF. Complexity of the self. New York: Guilford Press, 1987.

[29] Shear MK. Factors in the etiology and pathogenesis of panic disorder: revisiting the attachment-separation paradigm. Am J Psychiatry 1996; 153(7 Suppl): 125-36.

[30] Chorpita BF, Barlow DH. The development of anxiety: the role of control in the early environment. Psychol Bull 1998; 124: 3-21.

[31] Marazziti D, Dell'osso B, Catena Dell'Osso M, et al. Romantic attachment in patients with mood and anxiety disorders. CNS Spectr. 2007; 12(10): 751-6.

[32] Manassis, K. Child-parent relations: Attachment and anxiety disorders. New York: Cambridge University Press 2001.

[33] Dozier M, Stovall-McClough KC, Albus KE. Attachment and psychopathology in adulthood. In Cassidy J, Shaver P (Eds.), Handbook of attachment. $2^{\text {nd }}$ ed. New York: Guilford Press 2008; pp. 718-44.

[34] Shorey HS, Synder CR. The role of adult attachment styles in psychopathology and psychotherapy outcomes. Rev Gen Psychol 2006; 10: 1-20.

[35] Nakash-Eiskovits O, Dutra L, Westen D. Relationship between attachment patterns and personality pathology in adolescents. J Am Acad Child Adol Psychol 2002; 41: 1111-23.

[36] Manassis K, Bradley S, Goldberg S, Hood J, Swinson RP. Attachment in mothers with anxiety disorders and their children. J Am Acad Child Adolesc Psychiatry 1994; 33(8): 1106-13.

[37] Fraley RC. Attachment stability from infancy to adulthood: Metaanalysis and dynamic modeling of developmental mechanisms. Pers Soc Psychol Rev 2002; 6: 123-51.

[38] Grossmann KE, Grossmann K, Waters E, Eds. Attachment from infancy to adulthood: The major longitudinal studies. New York: Guilford Press 2005.

[39] Picardi A, Caroppo E, Toni A, Bitetti D, Di Maria G. Stability of attachment-related anxiety and avoidance and their relationships with the five-factor model and the psychobiological model of personality. Psychol Psychother 2005; 78: 327-45.

[40] Jackson MJ, Turkington D. Depression and anxiety in epilepsy. J Neurol Neurosurg Psychiatry 2005; 76(Suppl 1): i45-7.

[41] Roisman GI, Holland A, Fortuna K, Fraley C, Clausell E, Clarke A. The Adult Attachment Interview and self-reports of attachment style: an empirical reapprochement. J Pers Soc Psychol 2007; 92: 678-97.

[42] Bowlby J. A secure base. New York: Basic Books 1988.

[43] Fonagy P, Luyten P. A developmental, mentalization-based approach to the understanding and treatment of borderline personality disorder. Dev Psychopathol 2009; 21: 1355-81. 
[44] Fonagy P. Attachment Theory and Psychoanalysis. New York: Other Press 2001.

[45] Holmes J. In search of the secure base. London: Routledge 2001.
[46] Guidano VF. The self in process. New York: Guilford Press 1991.

[47] Byng-Hall J. Creating a family science base: Some implications of attachment theory for family therapy. Fam Proc 1995; 34: 45-58.

Received: January 07, 2013

Revised: July 28, 2013

Accepted: August 07, 2013

(C) Picardi et al.; Licensee Bentham Open.

This is an open access article licensed under the terms of the Creative Commons Attribution Non-Commercial License (http://creativecommons.org/licenses/by-nc/3.0/) which permits unrestricted, non-commercial use, distribution and reproduction in any medium, provided the work is properly cited. 